\title{
MicroscopyPioneers Pioneers in Optics: Étienne-Jules Marey and Paul Gottlieb Nipkow
}

\author{
Michael W. Davidson \\ National High Magnetic Field Laboratory, Florida State University, Tallahassee, FL 32306 \\ davidson@magnet.fsu.edu
}

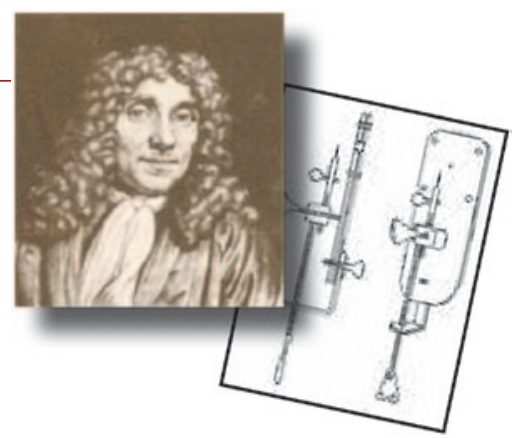

\section{Étienne-Jules Marey \\ (1830-1904)}

Étienne-Jules Marey was born in Beaune, France, on March 5, 1830, to a wine merchant and a teacher. To satisfy his father's wishes, he decided to study medicine, attending the Faculty of Medicine in Paris. Marey's fascination with both animals and mechanics inspired him to concentrate on physiology, and it was during his investigations of this field that he became involved in optics.

Due to a personal opposition to vivisection, Marey was inspired to find new ways to study subjects. Because his primary interest lay in developing an understanding of the motion of bodies, Marey invented a number of devices that could record movements.

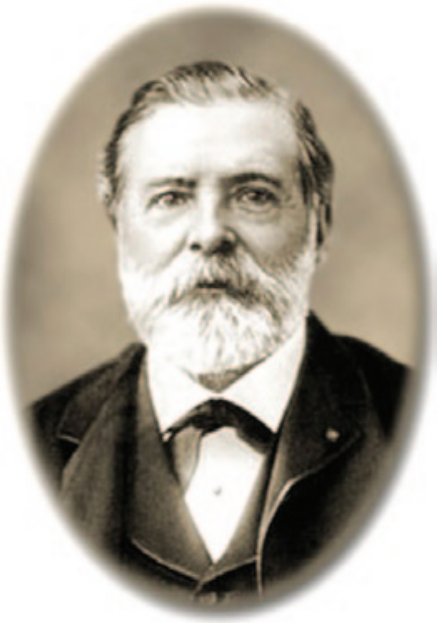

His initial graphical methods enabled him to examine the way humans and horses walk, as well as how birds fly. Limitations to the techniques, however, motivated Marey to consider different approaches, and he was intrigued by the possibility of adapting the nascent photography methods of the period to meet the needs of his scientific research. At the time, Eadweard Muybridge had already begun his photographic experiments involving horses in motion, and when Marey saw his images in the journal La Nature in 1879 , he began a correspondence with the photographer.

Marey asked Muybridge to apply his technique to the flight of birds but was unsatisfied by the results obtained. Some of the most questionable aspects that Marey found in Muybridge's work involved his basic setup, which consisted of a series of cameras that were placed parallel and adjacent to the subject's path of movement. Marey realized that the use of a different camera to record each image meant that there was no single point of reference from which changes in position could be assessed. Moreover, accurately measuring the gaps of time between movements was problematic, and, therefore, the representation of motion that was achieved was incomplete. Over the next twenty years Marey carried out a series of efforts to correct these perceived shortcomings and to make the photography of motion a more scientific endeavor.

By 1881, Marey was a professor at the College de France and was provided enough funding to establish a laboratory dedicated to physiological research. In this new setting, he developed a variety of investigative methods, the most basic of which involved recording multiple images of a subject's motions on a single camera plate. Over time he refined this technique and was capable of taking 12 pictures per second using a photographic "gun," which looked similar to a rifle and is commonly considered the first movie camera. Following the release of improved photographic film by George Eastman in 1885, Marey was able to vastly increase the photographic gun's exposure speed to 60 images per second, greatly improving the quality of his motion pictures and essentially laying the foundations of modern cinematography.

\section{Paul Gottlieb Nipkow (1860-1940)}

Paul Gottlieb Nipkow was a German engineer and inventor who proposed the world's first electromechanical television system. He was born on August 22, 1860, in Lauenberg, Germany, and studied at the University of Berlin. It was during his time as a student there that he developed the idea he is best known for.

While some nineteenth century scientists, such as Guglielmo Marconi, concentrated on transmitting audio signals, Nipkow was interested in the notion of transmitting a visual signal. At the young age of 23 , he proposed a method that was capable of the task and received a patent for his invention. What Nipkow referred to as an electric

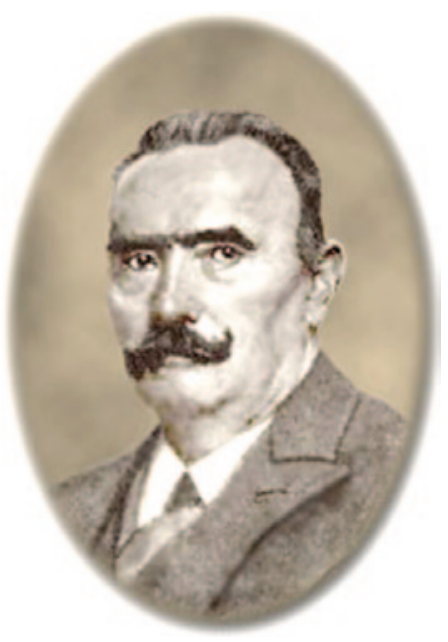
telescope was actually the forerunner of modern television. The innovative system was based on a simple device known as the Nipkow disk.

The Nipkow disk was composed of either metal or cardboard, perforated with a series of square holes arranged in a spiral pattern, with each hole slightly nearer to the center than the previous hole. As the Nipkow disk was rotated rapidly in front of a light-sensitive selenium cell on which a lens formed an image, an intense light was shined through the holes. Though each hole essentially "scanned" a small section of the image, together they created a full replica of the image in a single rotation of the disk. The image could then be converted into an electrical signal by connecting the selenium photoelectric cell to an electric current. 


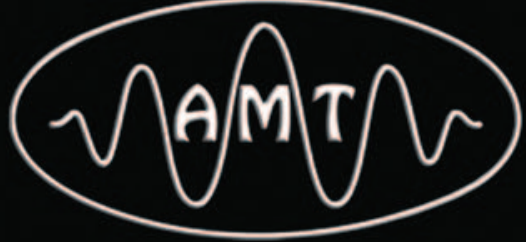

High Definition Digital

TEM Cameras with

1 to 16 Megapixels
- AMT SOLUTIONS

- Life Science Cameras

- Material Science Cameras

- Easy To Use Software

- Reliability and Services

- TEM Integration

- Extensive Support
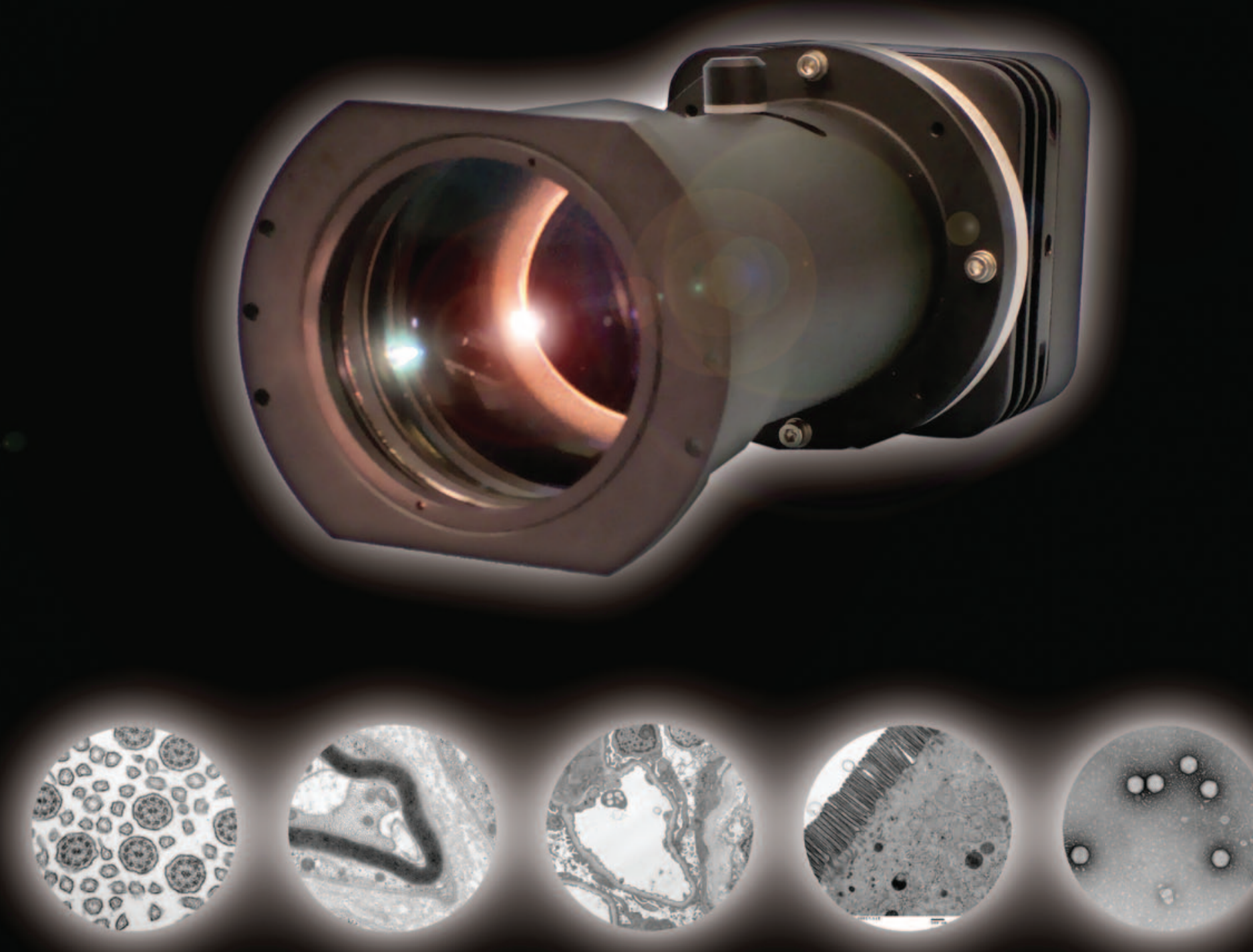
The image was reproduced at a receiver where an identical disk was synchronized with the first in front of a lamp whose brightness changed according to the received signal.

Nipkow once used his device to transmit a visual image from London to Paris, but the system was never developed for commercial use. Ironically, at the time, investors could not foresee a practical use for it, and, therefore, Nipkow received little recognition during his lifetime for the feat. He spent most of his life as a railway engineer and died in Berlin, Germany, on August 24, 1940. However, Nipkow paved the way for future developments in television, and the horizontal-scanning method he first conceived continues to be an essential element in modern-day electronics.

The Nipkow disk is currently used extensively in reflected light confocal scanning microscopy to produce images that can be viewed in real time through the microscope eyepieces. The rotating, perforated disk fulfills the requirements of both point illumination and detection when a modulated light beam is passed through the numerous holes drilled along a spiral. Each illuminated pinhole on the spinning disk is imaged by the objective to a diffraction-limited spot on the specimen. Light reflected from the specimen can be observed in the eyepieces (or with a CCD camera system) after it has passed back through a conjugate pinhole in the Nipkow disk. Several thousand points are simultaneously illuminated on the disk to mimic the effect of several thousand confocal microscopes running in parallel. The rapidly spinning disk fills spaces between the holes to create a real-time confocal image.

\section{PELCO ${ }^{\circ}$ Silicon Nitride \&} Silicon Dioxide Membranes

Next Generation SiN TEM Support Films

- Robust and clean $8,15,50 \& 200 \mathrm{~nm}$ $\mathrm{SiN}$ substrates

- $\varnothing 3.0 \mathrm{~mm}$ frame

- EasyGrip ${ }^{\text {Tw }}$ edges

- Free from debris

- Super flat 8, 15 and $40 \mathrm{~nm}$ Silicon Dioxide Substrates

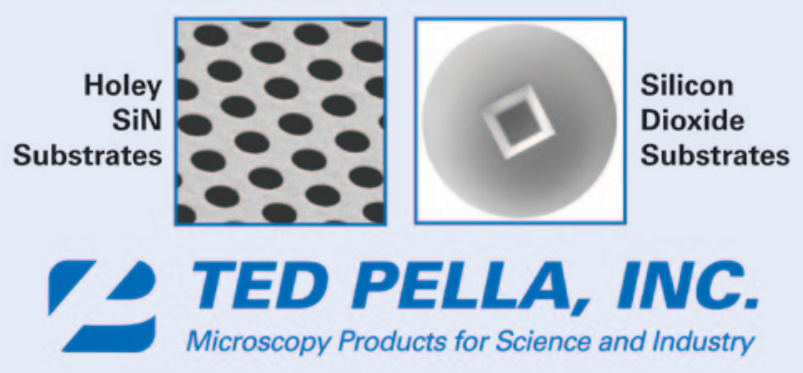

sales@tedpella.com 800-237-3526 www.tedpella.com

\section{lumencor a powerful, white-light, solid-state illuminator why buy a lamp when you can have a light engine?}

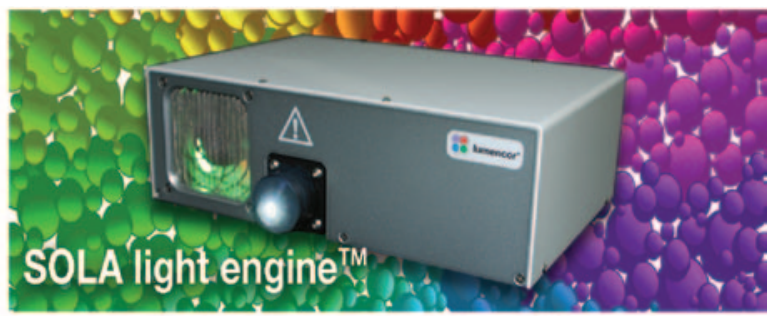

Solid State Sources, the Power of an Arc Lamp and

- Powerful, stable white light

- Minimal heat generation

- Illumination uniformity

- Short warm-up time

- Millisecond switching times

- Long life > 15,000 usable hours

- Off-the-shelf configurations

- Use with any microscopy software in the marketplace

- Couples to all major brands of microscope via 3mm LLG

- Integrates with existing filter cubes and hardware configurations specific outputs are a function of instrument parameters - results will vary

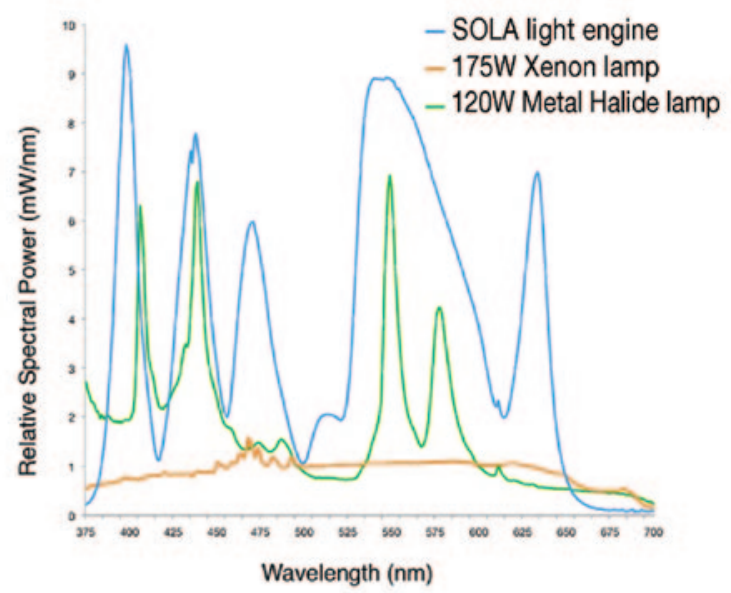




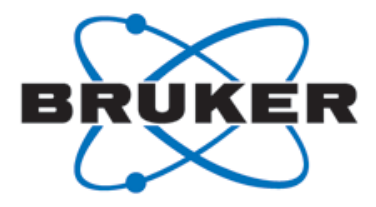

\section{Count on Us! Best EDS Performance with the NEW Slim-line XFlash 6}

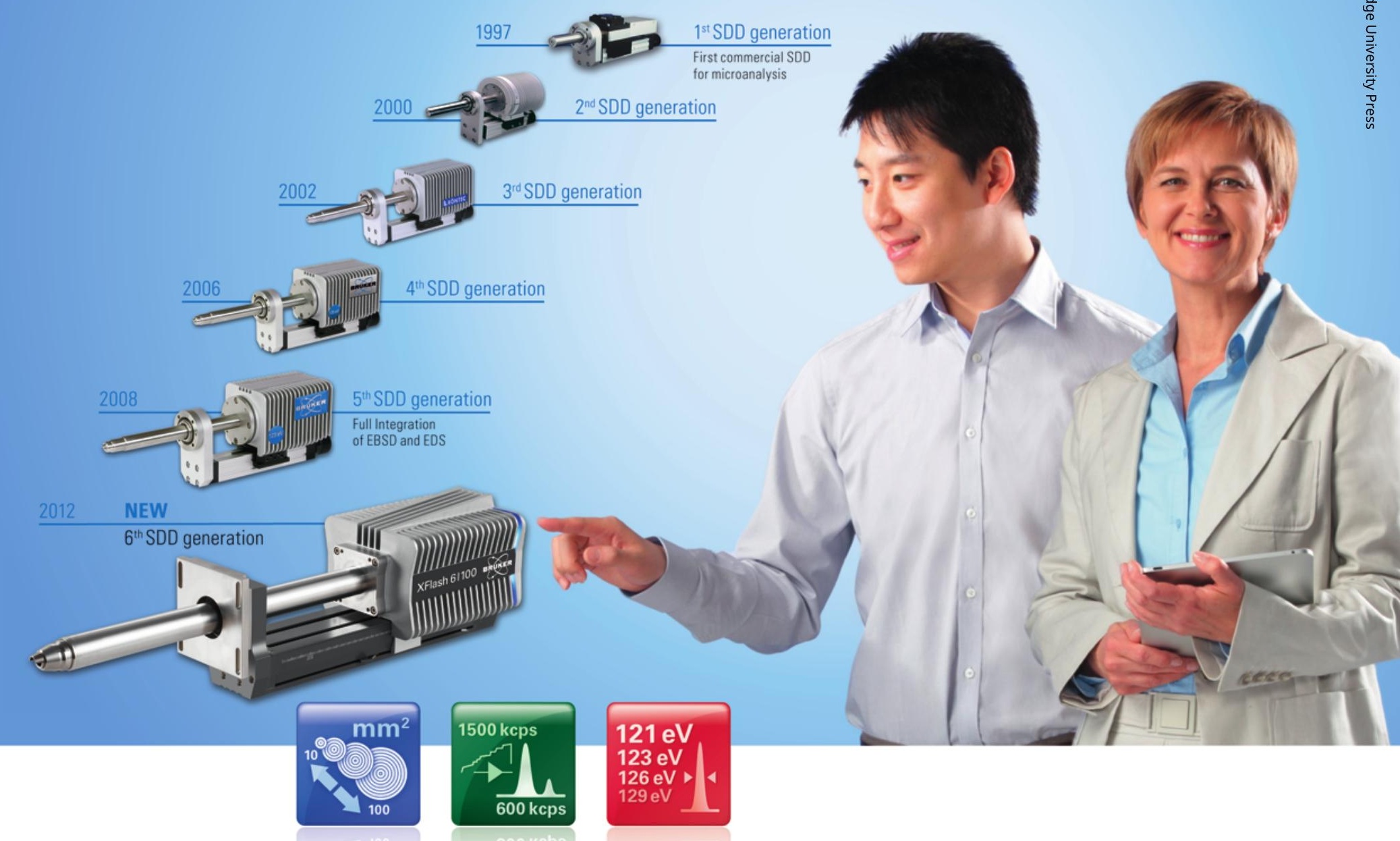

You can count on the NEW XFlash ${ }^{\circledR}$ SDD generation:

- Best solid angle - optimum geometry and active areas from $10 \mathrm{~mm}^{2}$ to $100 \mathrm{~mm}^{2}$

- Best throughput - up to $600,000 \mathrm{cps}$ output at 1,500,000 cps input

- Best energy resolution - $121 \mathrm{eV}$ at $\mathrm{Mn} \mathrm{K} \alpha, 47 \mathrm{eV}$ at $\mathrm{F} \mathrm{K \alpha}, 38 \mathrm{eV}$ at C K $\alpha$ (FWHM, exceeds ISO 15632:2002 requirements) www.bruker.com 